\title{
At first glance, transparency enhances assimilation
}

\author{
Arno Koning, Charles M M de Weert, Rob van Lier \\ Nijmegen Institute for Cognition and Information, Radboud University Nijmegen, PO Box 9104, \\ 6500 HE Nijmegen, The Netherlands; e-mail: a.koning@nici.ru.nl \\ Received 22 October 2007, in revised form 19 March 2008
}

\begin{abstract}
We investigated the role of transparency, perceptual grouping, and presentation time on perceived lightness. Both transparency and perceptual grouping have been found to result in assimilation effects, but only for ambiguous stimulus displays and with specific attentional instructions. By varying the presentation times of displays with two partly overlapping transparent E-shaped objects, we measured assimilation in unambiguous stimulus displays and without specific attentional instructions. The task was to judge which of two simultaneously presented E-shaped objects was darker. With unrestrained presentation times, if a transparency interpretation was possible, assimilation was not found. Inhibiting a transparency interpretation by occluding the local junctions between the two E-shaped objects, did lead to assimilation. With short presentation times, if a transparency interpretation was possible, assimilation was now also found. Thus, we conclude that, although transparency appears to enhance assimilation, with unambiguous stimulus displays and without specific attentional instructions, perceptual grouping is more important for assimilation to occur.
\end{abstract}

\section{Introduction}

It is a well known phenomenon that a grey patch viewed against a dark background is perceived as lighter than the same grey patch viewed against a light background. This lightness illusion is called the simultaneous contrast effect. Another class of lightness illusions concerns assimilation. That is, whereas contrast effects lead to a perceptual difference of similar (or equal) luminances, assimilation effects lead to a perceptual similarity of different luminances. Physiological models that emphasise lateral inhibition of cells (either in the retina or in the visual cortex) have been fairly successful in explaining contrast effects, whereas assimilation effects seem to require a more higherlevel account. In the past decades more and more studies have shown that higher-level aspects such as 3-D shape (Adelson 1993), layout of the scene (Gilchrist 1977), curvature (Knill and Kersten 1991), illusory contours (eg van Lier 2002; van Tuijl and de Weert 1979), figure-ground segmentation (eg de Weert and van Kruijsbergen 1997), voluntary attention (Tse 2005; Tse et al 2006), and perceptual grouping (Agostini and Proffitt 1993; van Lier and Wagemans 1997) play an important role in both types of lightness illusions.

An example of the importance of perceptual grouping on perceived lightness is given by the study by Agostini and Proffitt (1993). In their stimulus displays, a homogeneous blue background was filled with black and white disks as well as two (identical) grey ones. When the disks were stationary, the grey disks appeared to be identical to each other. When the disks started moving, however, the grey disks no longer appeared to be of the same lightness: the grey disk that was grouped with the black disks by common fate was perceived to be lighter than the grey disk that was grouped by common fate with the white disks. This simultaneous contrast effect due to perceptual grouping (ie common fate) cannot be explained by lateral inhibition, but is in line with the anchoring model of lightness perception of Gilchrist et al (1999). 
Perceptual grouping and perceived lightness have not solely been studied in the context of contrast effects but also in the context of assimilation effects. For example, von Bezold (1876) was the first to notice that the perceived colour of an element depended on the way in which it was perceptually grouped with other, differently coloured, elements. More specifically, the colour of the element appeared to be assimilated with the colour of the other elements with which it was grouped perceptually. Fuchs (1923) examined this phenomenon more systematically and argued for the role of good form (or Prägnanz in Gestalt terms) as mediating the effect of perceived colour assimilation. For example, in figure 1a, if the central orange dot is considered to be part of a red cross $(\times)$, it appears to look more reddish. If the same orange dot is considered to be part of a yellow cross $(+)$, the orange dot appears to be more yellow. More recently, van Lier and Wagemans (1997) constructed displays that were ambiguous with respect to the way in which the chromatic elements could be grouped (ie the elements could be grouped on the basis of regularity or relative proximity). By contrasting the Gestalt principles of regularity and proximity, van Lier and Wagemans found that colour assimilation of a target element depended on the way in which this target element was grouped with other, differently coloured, elements. Thus, in both Fuchs's early investigations and the study by van Lier and Wagemans, colour assimilation of an element was found when it was grouped with other, differently coloured, elements.

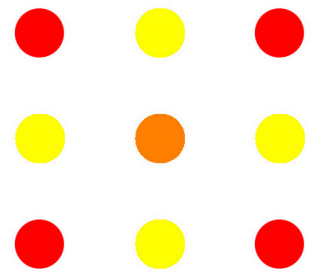

(a)

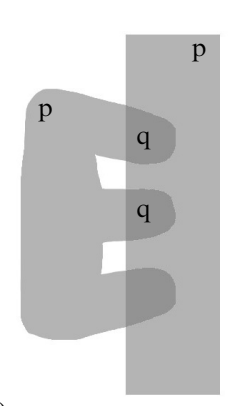

(c)

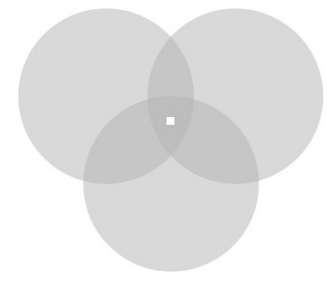

(b)

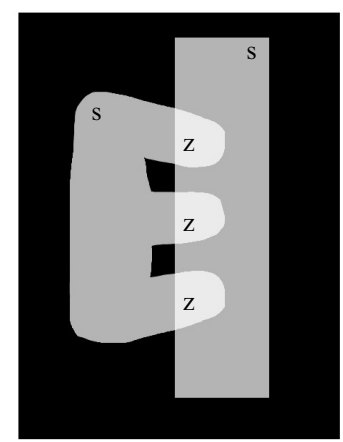

(d)

Figure 1. (a) Demonstration by Fuchs (1923) that perceptual grouping involves colour assimilation. If the central orange dot is perceptually grouped with the red dots into an ex $(\times)$, it appears to look more reddish, whereas if the same dot is perceptually grouped with the yellow dots into a cross $(+)$, the orange dot appears to look more yellowish [shown in colour on the Perception website http://dx.doi.org/10.1068/p6051]. (b) Demonstration by Tse (2005) that attention plays a role in perceived lightness of transparent surfaces. Focusing on the central white dot while covertly attending to one of the disks causes the attended disk to be perceived as darker than the remaining two disks. (c) Example of our initial observation that a transparent E-shaped object partly overlapping a rectangle can be perceived as darker than the rectangle when the entire display is shown briefly (same letters indicates same luminance). (d) Reversing the polarity of (c) also reverses the illusion in that now the E-shaped object is judged to be lighter than the rectangle. 
In the experiment by Fuchs (1923), for assimilation to occur one needs to attend to a specific organisation. Attention thus appears to play an important role in the interplay between perceptual grouping and assimilation. Recently, Tse (2005) emphasised the role of covert attention in the process of assimilation. Figure $1 \mathrm{~b}$ shows the configuration used by Tse and is easily interpreted as comprising three partly overlapping transparent (achromatic) disks. Fixating the centre dot while covertly attending one of the disks results in assimilation with the attended disk being perceived as darker than the other two disks. Now, in the larger context of perceptual grouping and perceived lightness the following could be the case. In displays, such as the ones used by Agostini and Proffitt (1993), grouping occurs by common fate of the visual elements, is almost immediate the moment the disks start moving, and there is no need for specific (attentional) instructions. The result of this is the contrast effect that Agostini and Proffitt found. Assimilation might occur in more ambiguous displays in which attention plays a role as well (eg Fuchs 1923; Tse 2005). This is in line with the suggestion by Agostini et al (2006) that assimilation tends to occur when perceptual grouping is ambiguous or unstable, whereas a contrast effect occurs when perceptual grouping is stable.

In Tse's (2005) example, the emphasis on covert attention comprises two essential aspects if assimilation is to occur. First, the observer must not make any eye movements. Second, attention needs to be allocated to one of the three identical disks. Eye movements can be easily prevented by using brief presentations of stimuli; attention can also be directed by manipulating the way different surfaces are perceptually grouped. Therefore, an experiment that incorporates both of these aspects will be able to more closely investigate the role of transparency in perceptual grouping and assimilation. Consider now the configuration shown in figure $1 \mathrm{c}$ that comprises an E-shaped object partly overlapping a grey rectangle. Due to the local X-junctions between these two objects, a transparency interpretation is facilitated. The grey parts indicated with the letter $\mathrm{p}$ have a higher luminance than the grey parts indicated with the letter $\mathrm{q}$. That is, the parts indicated by $\mathrm{p}$ are lighter than the parts indicated by q. In our initial observations, we noticed that when these displays were shown briefly, the E-shaped object appeared to be darker than the rectangle. Clearly, the parts indicated by the letter $\mathrm{q}$ are grouped with the left E-shaped object and initially this seems to lead to an assimilation effect. One can test this assimilation effect by looking away from the figure and then after only briefly glancing at figure 1c, looking away again. Furthermore, in figure 1d the polarity of the background is reversed and the parts indicated by the letter s have a lower luminance than the parts indicated by the letter z. When this configuration is viewed briefly, the E-shaped object appears to be lighter than the rectangular shape. These examples seem to indicate that a brief presentation of two surfaces that allows for a transparency interpretation can lead to assimilation without instructions regarding a specific organisation or covert attention. In order to investigate the role of transparency, perceptual grouping, and presentation time in assimilation, we will use stimuli similar to the displays shown in figure 1c in an experiment in which the relative lightness of two simultaneously presented objects has to be judged. On the basis of previous studies (eg Agostini et al 2006; Fuchs 1923; van Lier and Wagemans 1997; Tse 2005) and our initial observations we expect that, when perceptual grouping is triggered by transparency cues, assimilation effects can be found as well, especially when briefly viewing the stimuli. 


\section{Experiment}

\subsection{Methods}

2.1.1 Participants. Nine undergraduate students of psychology at the Radboud University Nijmegen, aged $19-23$ years, performed the experiment. The students were given course credit or were paid for their time. All participants reported normal or corrected-to-normal vision.

2.1.2 Stimuli. Two E-shaped objects were produced which were used to create four different conditions (see figure 2 and also table 1 for the different luminance values used). In the first condition, the transparency condition (figure 2a), the parts indicated by the letter $\mathrm{q}$ had a slightly lower luminance than the surrounding parts indicated by the letter $\mathrm{p}$. In this case a transparency interpretation was likely. To investigate whether transparency in this type of display is necessary for assimilation to occur, a control is needed. Therefore, for the second condition, the occlusion condition (figure $2 b$ ), a transparency interpretation was inhibited by adding a noise band to occlude the local $\mathrm{X}$-junctions between the two E-shaped objects. That is, local X-junctions between two surfaces are considered to be crucial for triggering a transparency interpretation. The third condition was considered the baseline condition (figure $2 \mathrm{c}$ ) as now the overlapping parts, indicated with the letter a, had the same luminance as the background. Finally, in the baseline/occlusion condition (figure 2d), a noise band was again placed such that the local junctions between the parts a and $\mathrm{p}$ were occluded.

In addition to these four conditions, the luminance relations between the E-shaped objects were also varied: both parts had the same luminance, or the left E-shaped object had a lower luminance, or the right E-shaped object had a lower luminance. As a result, 12 unique images were created: 4 configurations (see figure 2 ) $\times 3$ luminance relations between the two E-shaped objects. In addition, each image was also mirrored left - right, yielding a total of 24 trials for the experiment.

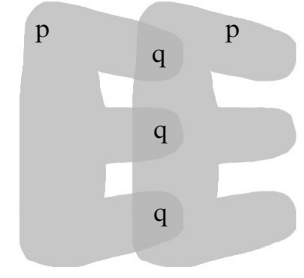

(a)

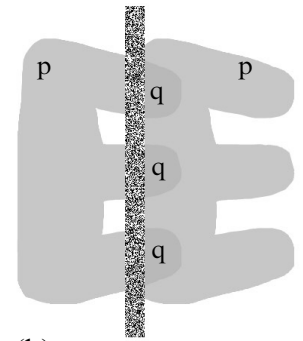

(b)

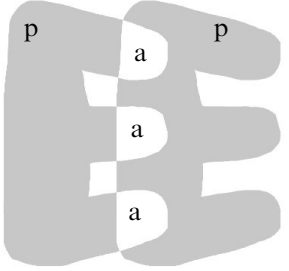

(c)

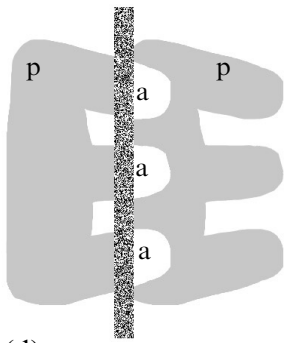

(d)

Figure 2. Examples of the stimuli used in the experiment (same letters indicate same luminance). (a) The transparency condition in which the parts indicated by the letter q have a lower luminance than the parts indicated by the letter p. (b) The occlusion condition in which a noise band is added to occlude the local X-junctions between the two E-shaped objects thereby inhibiting a transparency interpretation. (c) The baseline condition in which the areas indicated with the letter a have the same luminance as the background. (d) The baseline/occlusion condition in which a noise band is added to occlude the local junctions between the two E-shaped objects.

Table 1. Luminance values of the different parts of the stimulus displays.

Part of the stimulus display

Luminance/ $\mathrm{cd} \mathrm{m}^{-2}$

To-be-judged parts ( $\mathrm{p}$ in figure 2) have the same luminance 
2.1.3 Procedure. The experiment was conducted at the Radboud University Nijmegen in a darkened room with the only light being that emitted by the monitor. The stimuli were presented with SuperlabPro (Cedrus Inc.) on a Pentium IV computer and a 19 inch monitor. A beep $(250 \mathrm{~Hz})$ was used to signal the onset of the trial. The experiment consisted of two procedures with different presentation times. In one part (unrestrained presentation times), after the beep, the stimulus was shown and remained on the screen until the participant responded, after which a blank screen appeared for $750 \mathrm{~ms}$, thereby completing a single trial. In the other part (short presentation times), after the beep, the stimulus was presented for $100 \mathrm{~ms}, 200 \mathrm{~ms}$, or $300 \mathrm{~ms}$, followed by a blank screen. The order of these two procedures with different timelines (unrestrained presentation times first versus short presentation times first) was counterbalanced across participants. When presentation times were unrestrained, the 24 trials were randomly shown in a single block and ten blocks were administered in total, with a short break after the fifth block. Thus, in total 240 trials were shown in this part of the experiment. When short presentation times were used, the 24 trials were presented for $100 \mathrm{~ms}, 200 \mathrm{~ms}$, or $300 \mathrm{~ms}$ in a single block. Thus each block comprised 72 trials. In total, four blocks (totaling 288 trials) were shown, with a short break halfway. Combining both presentation times (unrestrained and short), each participant thus received 528 trials.

The experiment was preceded by a short explanation in which the participant was told that judgments had to be made which E-shaped object was darker. In between the two procedures a short break was inserted and additional instructions were given based on the presentation sequence (unrestrained presentation times or short presentation times). Participants responded by pressing the left button on a response box (Cedrus Inc.) if they found the left E-shaped object darker, and pressing the right button for the right E-shaped object. The entire experiment was performed in one session and took about $35 \mathrm{~min}$ to complete.

\subsection{Results}

Preliminary analyses revealed no differences between the three different presentation times $(100 \mathrm{~ms}, 200 \mathrm{~ms}$, and $300 \mathrm{~ms})$ when the stimuli were presented for a short time; therefore the data were pooled over this variable. In addition, the data for three luminance relations between the two E-shaped objects were pooled. Next, for each participant and for each condition, the average number of times the left E-shaped object was judged to be darker than the right E-shaped object was computed. Notice that our decision to use a darker rating for the left E-shaped object (the left object as shown in figure 2) as a measure of assimilation instead of the right E-shaped object is arbitrary; the main focus is on the influence of transparency, perceptual grouping, and presentation time on assimilation effects. Naturally, all aspects of the two stimuli and the task concerning left and right have been balanced.

One-sample $t$-tests were performed to examine whether the number of times the left E-shaped object was judged to be darker differed significantly from 0.5. That is, when no differences were expected, the average number of times the left E-shaped object was judged darker than the right E-shaped object was not expected to deviate significantly from 0.5 . Table 2 shows all $t$-values, and figure 3 shows a plot of mean judgments that the left E-shaped object was darker than the right E-shaped object as a function of the four different kinds of displays and presentation times.

The results were as expected. First, in the transparency condition the two E-shaped objects were equally often judged to be darker $(p>0.2)$ when the presentation times were unrestrained. When the presentation times were short, the left E-shaped object was significantly more often judged to be darker than the right E-shaped object $\left(t_{8}=5.77\right.$, $p<0.001)$. In the occlusion condition, the left E-shaped object was consistently judged 
Table 2. $t$-Values of the one-sample $t$-tests (test value $=0.5$ ) for all conditions and both unrestrained and short presentation times.

\begin{tabular}{lccccc}
\hline \multirow{2}{*}{ Condition } & \multicolumn{2}{c}{ Unrestrained presentation times } & & \multicolumn{2}{c}{ Short presentation times } \\
\cline { 2 - 3 } \cline { 5 - 6 } & $t$-value & Significance, 2-tailed & & $t$-value & Significance, 2-tailed \\
\hline Transparency & 1.14 & 0.285 & & 5.77 & $\mathbf{0 . 0 0 1}$ \\
Occlusion & 20.38 & $\mathbf{0 . 0 0 1}$ & & 14.63 & $\mathbf{0 . 0 0 1}$ \\
Baseline & 1.55 & 0.160 & & 0.158 & 0.878 \\
Baseline/occlusion & -1.31 & 0.226 & & -1.44 & 0.189 \\
\hline
\end{tabular}

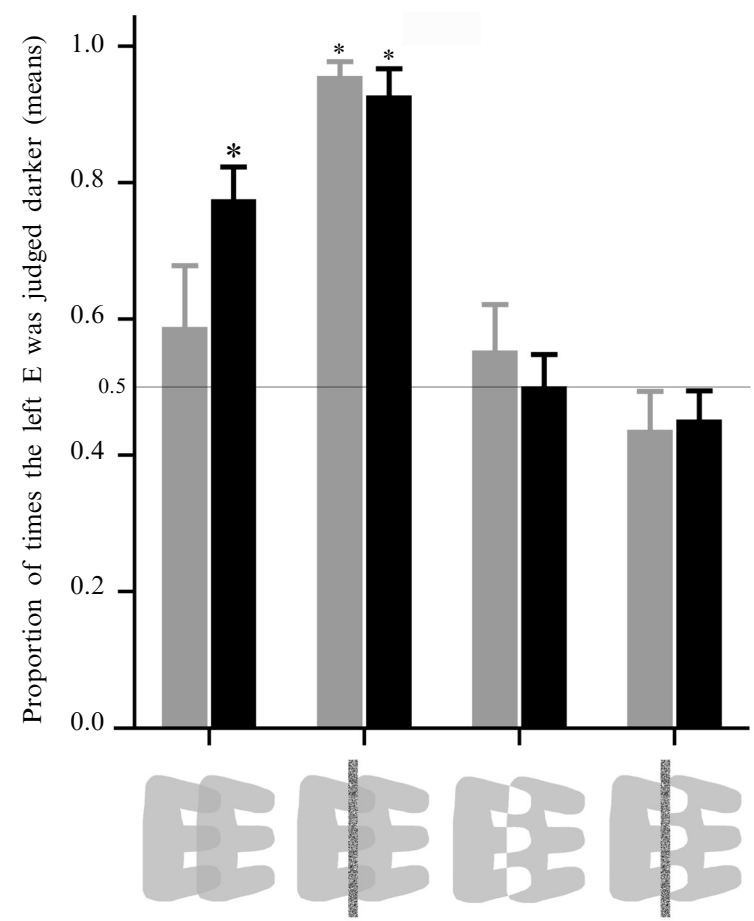

Figure 3. Experimental results. The mean proportions in which the left E-shaped object was judged darker than the right E-shaped object as a function of the four different stimulus configurations and presentation time. Error bars represent $1 \mathrm{SE}$. An asterisk indicates $p<0.05$.

to be darker than the right E-shaped object, both when the presentation times were unrestrained $\left(t_{8}=20.38, p<0.001\right)$, and when the presentation times were short $\left(t_{8}=14.63, p<0.001\right)$. Finally, in the two baseline conditions, the two E-shaped objects were equally often judged to be darker both when the presentation times were unrestrained and when the presentation times were short (all $p \mathrm{~s}>0.1$ ).

\section{General discussion}

Our aim in this study was to investigate the role of transparency, perceptual grouping, and presentation time in assimilation. Two partly overlapping E-shaped objects were used (achromatic and static stimuli-figures $2 \mathrm{a}$ and $2 \mathrm{~b}$ ) for which perceptual grouping was unambiguous, and no specific instructions regarding the direction of attention were given. In the case of unrestrained presentation times, an assimilation effect was found when a transparency interpretation was inhibited (owing to an occluding noise band-figure $2 b$ ), but not when a transparency interpretation was possible (owing to local transparency cues - figure 2a). When the same stimuli were presented for a short 
time, in both cases assimilation effects occurred. These effects conform to those found in previous studies in which assimilation effects due to perceptual grouping were reported (eg Fuchs 1923; van Lier and Wagemans 1997).

In the occlusion condition a transparency interpretation was inhibited by the occluding noise band and an assimilation effect was found in both parts of the experiment (unrestrained and short presentation times). This can be related to an inverse variant of the well-known Koffka ring (Koffka 1935). In figures $4 \mathrm{a}$ and $4 \mathrm{~b}$, the classic Koffka ring is shown. What is essential is that the ring has one luminance level and is positioned against a background with different luminance levels.

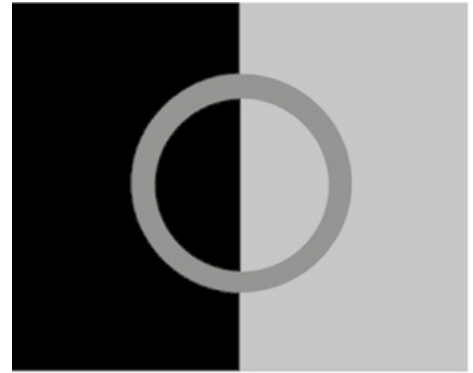

(a)

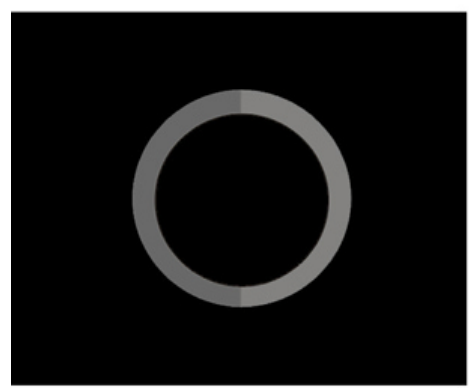

(c)

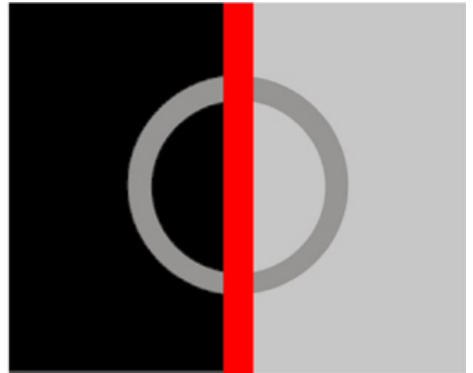

(b)

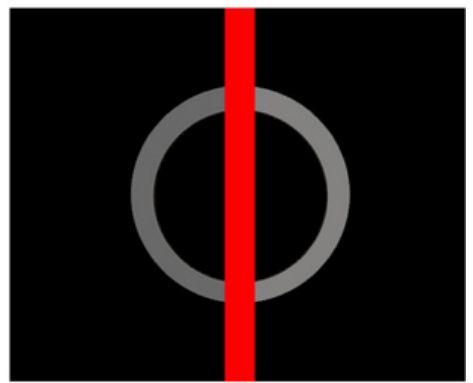

(d)

Figure 4. [In colour online.] (a) The Koffka ring. A homogeneously coloured ring is placed on a background that has two different luminance values. (b) Occluding both the ring and the luminance edge between the two halves of the background causes the ring to be no longer perceived as homogenous, but rather as two halves with a different perceived lightness. (c) The anti-Koffka ring. A ring, consisting of two halves that have a different luminance is placed on a homogenous background. (d) By occluding the local junctions between the two halves of the ring, assimilation causes the ring to be no longer perceived as comprising two halves, but rather as a homogenously coloured ring.

When both the ring and the luminance edge between the two halves of the background are covered by an occluding contour, the two halves of the ring are suddenly perceived as having a different lightness (see figure 4b). Alternatively, in our antiKoffka stimulus, shown in figure $4 \mathrm{c}$, the surround is homogeneous but the two halves of the ring differ in luminance. As a result, a luminance edge is clearly visible between these two halves. However, when an occluding contour is added that covers these luminance edges (figure 4d), direct evidence for the difference between the two halves is diminished, which seems to facilitate assimilation. Still, it seems reasonable to assume that the two halves in the anti-Koffka example appear similar in figure $4 \mathrm{~d}$ simply because of a higher luminance difference threshold (ie the threshold needed to perceive a luminance difference) as the two halves are no longer adjacent. That is, in the unoccluded condition (figure 4c) lateral inhibition of cells causes the edge between the two adjacent surfaces to be perceptually sharpened, whereas this sharpening is not 
there in the occluded condition (figure 4d). In a similar way in the occlusion condition (figure $2 \mathrm{~b}$ ), the E-shaped object and the q regions are not adjacent, thereby heightening the luminance difference threshold. Obviously, in the baseline and baseline/occlusion conditions (figure $2 \mathrm{c}$ and $2 \mathrm{~d}$ ), the occurrence of a perceptual sharpening (baseline) or its occlusion (baseline/occlusion) becomes irrelevant because of the much larger luminance differences. In addition to this explanation in terms of physical attributes of the stimulus displays, the assimilation effect found in the occlusion condition also seems to deal with more higher-level aspects: the E-shaped object in figure $2 \mathrm{~b}$ appears to take on the darker appearance of the small overlapping parts (the q regions in figure $2 b$ ), and not the other way round. With this apparent darkening of the E-shaped object, the resulting interpretation is that of an opaque, homogeneously coloured, E-shaped object in front of another E-shaped object.

Although the results of the occlusion condition suggest that transparency was not necessary for assimilation to occur in this experiment, an important finding here is that when transparent stimuli (as in Tse 2005) are shown briefly, assimilation can occur without explicitly directing attention towards a specific organisation (as in Fuchs 1923) or by means of covert attention (Tse 2005). In contrast, when presentation times were unrestrained, or when conditions for transparency were violated (as in the control condition-figure 2c), no assimilation was found. Although it could be argued that in the case of short presentation times an assimilation effect was found because sufficient time is needed before a luminance difference threshold for adjacent surfaces is lowered, this is rather unlikely given that earlier research has convincingly shown that this happens relatively quickly in around $80 \mathrm{~ms}$ (see, for example, Graham 1965). This is also reflected in the fact that our analyses did not show the presence of an effect of presentation time (we used presentation times of $100 \mathrm{~ms}, 200 \mathrm{~ms}$, and $300 \mathrm{~ms}$ ).

Instead, with regard to our findings in the transparency condition, we propose the following underlying mechanism. A first glance at a transparent stimulus enhances assimilation in a well-defined direction (eg in figure $2 \mathrm{a}$ the overlapping parts belong to the left E-shaped object) because of initial grouping and an additional attentional bias in the same direction (eg in figure $2 \mathrm{a}$ towards the resulting E-shape). This would be in line with the studies of Tse (2005) and Tse et al (2006) in which covertly attending one of three partly overlapping transparent surfaces (figure 1d) led to assimilation. More specifically, with regard to our experimental procedure, when presentation times are unrestrained and no specific attentional instructions are given, the participant can compare the two E-shaped objects, for example by moving his/her eyes, attending to both objects, before making a judgment. In this case the transparency condition will not lead to an assimilation effect. A further comment here concerns the perception of transparency itself in our displays. It could very well be that with short presentation times, owing to the above described assimilation effect, a transparency interpretation is not fully generated, even though such an interpretation is possible as a result of the local X-junctions.

In sum, in line with previous studies (eg Adelson 1993; Agostini and Proffitt 1993; Fuchs 1923; Gilchrist 1977; Knill and Kersten 1991; van Lier and Wagemans 1997; Tse 2005; Tse et al 2006), we found that perceptual grouping influences perceived lightness. More specifically, we show that, while at first glance, perceptual transparency seems to facilitate assimilation, it is rather perceptual grouping, triggered by the specific stimulus properties, that does the work.

Acknowledgments. This study was performed at the Radboud University Nijmegen. We would like to thank Pim Haerkens for his help in running the experiment and two anonymous reviewers for their helpful comments and suggestions to a previous draft of this paper. 


\section{References}

Adelson E, 1993 "Perceptual organization and the judgment of brightness" Science 2622042 - 2044 Agostini T A, Galmonte A C G, Righi G, 2006 "Achromatic contrast and assimilation: The role of perceptual organisation" Perception 35 Supplement, 181

Agostini T A, Proffitt D, 1993 "Perceptual organization evokes simultaneous lightness contrast" Perception $22263-272$

Bezold W von, 1876 The Theory of Color (Boston, MA: Prang)

Fuchs W, 1923 "Experimentelle Untersuchungen über die Änderung von Farben unter dem Einfluss von Gestalten (Angleichungserscheinungen)" [Experimental investigations on the change of colour under the influence of Gestalten] Zeitschrift für Psychologie 92 249-325

Gilchrist A L, 1977 "Perceived lightness depends on perceived spatial arrangement" Science 195 $185-187$

Gilchrist A L, Kossyfidis C, Bonato F, Agostini T A, Cataliotti J, Xiajun L, Spehar B, Annan V, Economou E, 1999 "An anchoring theory of lightness perception" Psychological Review 106 $795-834$

Graham C H, 1965 Vision and Visual Perception (New York: John Wiley and Sons)

Knill D, Kersten D, 1991 "Apparent surface curvature affects lightness perception" Nature 351 $228-230$

Koffka K, 1935 Principles of Gestalt Psychology (London: Lund Humphries)

Lier R van, 2002 "A double neon colour illusion" Perception 3131 - 38

Lier R van, Wagemans J, 1997 "Perceptual grouping measured by color assimilation: Regularity versus proximity" Acta Psychologica 9737 -70

Tse P U, 2005 "Voluntary attention modulates the brightness of overlapping transparent surfaces" Vision Research $451095-1098$

Tse P U, Caplovitz G P, Hsieh P J, 2006 "Microsaccade directions do not predict directionality of illusory brightness changes of overlapping transparent surfaces" Vision Research 46 3823-3830

Tuijl H F J M van, Weert C M M de, 1979 "Sensory conditions for the occurrence of the neonspreading illusion" Perception $8211-215$

Weert C M M de, Kruijsbergen N A W H van, 1997 "Assimilation: central and peripheral effects" Perception $261217-1224$ 


\section{PERTEPTION}

VOLUME 372008

www.perceptionweb.com

Conditions of use. This article may be downloaded from the Perception website for personal research by members of subscribing organisations. Authors are entitled to distribute their own article (in printed form or by e-mail) to up to 50 people. This PDF may not be placed on any website (or other online distribution system) without permission of the publisher. 